Article

\title{
Structure-Based Pharmacophore Design and Virtual Screening for Novel Tubulin Inhibitors with Potential Anticancer Activity
}

\author{
Yunjiang Zhou ${ }^{1}$, Bin $\mathrm{Di}^{2, *}$ and Miao-Miao $\mathrm{Niu}^{2, *}$ (1) \\ 1 State Key Laboratory of Natural Medicines, School of Basic Medicine and Clinical Pharmacy, \\ China Pharmaceutical University, Nanjing 210009, China \\ 2 Department of Pharmaceutical Analysis, China Pharmaceutical University, Nanjing 210009, China \\ * Correspondence: dibin@cpu.edu.cn (B.D.); niumm@cpu.edu.cn (M.-M.N.); Tel.: +86-25-8327-1080 (M.-M.N.)
}

Received: 9 August 2019; Accepted: 29 August 2019; Published: 1 September 2019

\begin{abstract}
Tubulin inhibitors have been considered as potential drugs for cancer therapy. However, their drug resistance and serious side-effects are the main reasons for clinical treatment failure. Therefore, there is still an urgent need to develop effective therapeutic drugs. Herein, a structure-based pharmacophore model was developed based on the co-crystallized structures of the tubulin with a high resolution. The model including one hydrogen-bond acceptor feature, two aromatic features, and one hydrophobic feature was further validated using the Gunner-Henry score method. Virtual screening was performed by an integrated protocol that combines drug-likeness analysis, pharmacophore mapping, and molecular docking approaches. Finally, five hits were selected for biological evaluation. The results indicated that all these hits at the concentration of $40 \mu \mathrm{M}$ showed an inhibition of more than 50\% against five human tumor cells (MCF-7, U87MG, HCT-116, MDA-MB-231, and HepG2). Particularly, hit 1 effectively inhibited the proliferation of these tumor cells, with inhibition rates of more than $80 \%$. The results of tubulin polymerization and colchicine-site competition assays suggested that hit 1 significantly inhibited tubulin polymerization by binding to the colchicine site. Thus, hit 1 could be used as a potential chemotherapeutic agent for cancer treatment. This work also demonstrated the potential of our screening protocol to identify biologically active compounds.
\end{abstract}

Keywords: tubulin polymerization; pharmacophore modeling; molecular docking; cancer therapy

\section{Introduction}

Cancer is a fatal disease caused by uncontrolled cell proliferation, which has led to the deaths of 9.6 million people in 2018, and 18.1 million cases are newly diagnosed [1]. Microtubules (MTs) consist of typically 13 or 14 parallel protofilaments arising from the end-to-end aggregation of the tubulin $\alpha / \beta$-dimers [2]. MTs play a number of significant and diverse roles in all eukaryotic cells, such as cell motion, mitosis, and intracellular organelle transport [3]. By inhibiting the polymerization of tubulin or promoting microtubule depolymerization, most microtubule interfering agents (MIAs) show a very good therapeutic effect toward various cancer cells [4-6]. Therefore, microtubules can be an excellent target in the process of anticancer drugs design.

Most tubulin inhibitors bind to one of the three characterized tubulin ligand sites including taxol, vinca, and colchicine sites $[7,8]$. Among these sites, only drugs that interact with the colchicine site on tubulin can inhibit the process of angiogenesis (formation of new blood vessels) [8-14]. Currently, various types of colchicine binding site inhibitors (CBSIs) have been developed [15-20]. However, there are no FDA-approved drugs of this class on the market, because of their toxicity. For example, colchicine can cause impairment of renal and gastrointestinal functions and has a direct toxic action on myocardial 
cells [21-23]; ZD6126, a colchicine analogue, has significant cardiotoxicity even at normal doses [24-26]; fosbretabulin has many side effects, such as tumor pain, lymphopenia, blood pressure, and heart rate changes $[27,28]$. In addition, most CBSIs show low water solubility and poor pharmacokinetic properties [29]. Therefore, there is an urgent need to develop effective therapeutic drugs.

In our study, we used a combination of drug-likeness analysis, pharmacophore mapping, and molecular docking studies to find novel tubulin inhibitors. The pharmacophore model was constructed based on the two crystal structures of the tubulin with a high resolution. After validation by the Gunner-Henry score method, the model was used as a 3D query to screen potential drug-likeness molecules from the Specs database. The retrieved molecules were further filtered by molecular docking experiments. Finally, five structurally diverse hits were identified as potential leads for biological testing.

\section{Results and Discussion}

\subsection{Pharmacophore Modeling}

To obtain all available chemical and structural information on the inhibitor binding of the tubulin, a pharmacophore model was generated using a structure-based modeling approach, based on the four crystal structures of the tubulin (Table 1). This model was composed of four features (Figure 1): One hydrogen-bond acceptor feature (F1: Acc), two aromatic features (F2 and F3: Aro), and one hydrophobic feature (F4: Hyd). These features represented essential interaction points of the inhibitor binding of the tubulin: (i) Two Acc and Aro features (F1 and F2) corresponding to Leu240, Ala248, Leu253, and water molecules; (ii) two Aro and Hyd features (F3 and F4) corresponding to Met257, Ala314, Val181, and Lys350.

Table 1. Basic information of receptor-ligand complexes of the tubulin from the Protein Data Bank (PDB) database.

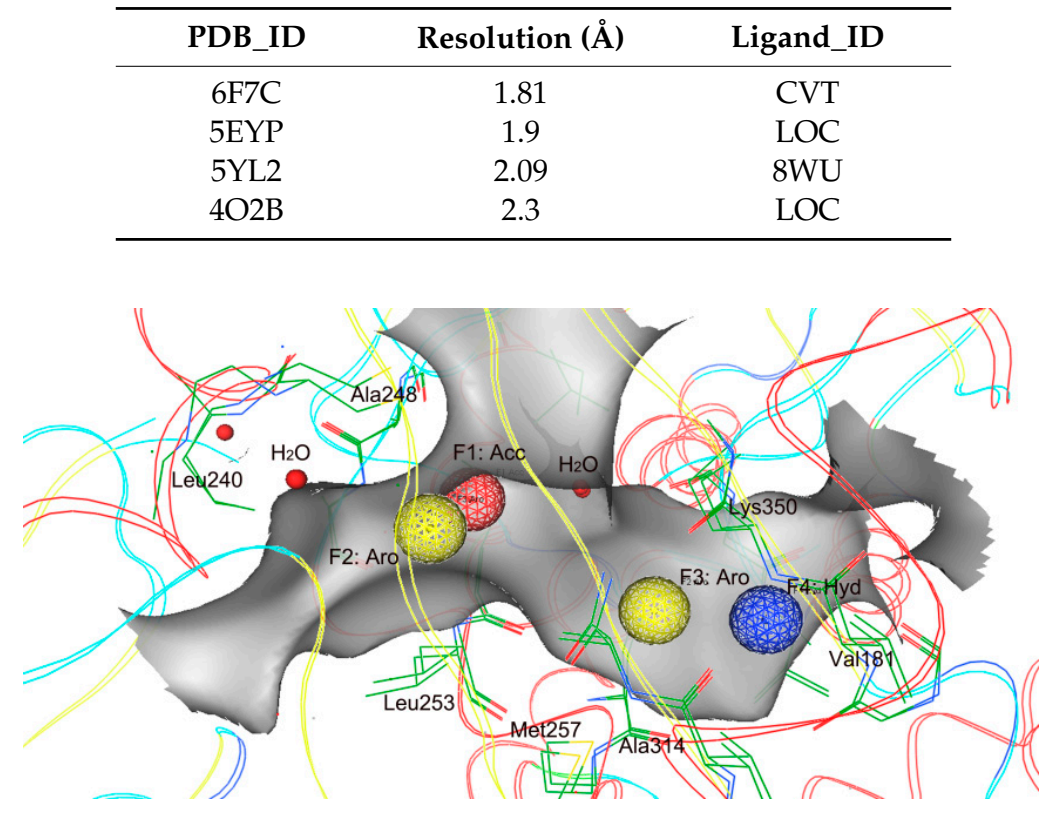

Figure 1. Generated pharmacophore model. Pharmacophore features are color-coded: Red, one hydrogen-bond acceptor feature (F1: Acc); yellow, aromatic features (F2 and F3: Aro); blue, one hydrophobic feature (F4: Hyd). The protein backbone and active site residues (green) are shown in line form; red balls mean water molecules; a gray pocket represents the shape of the binding site in tubulin. 


\subsection{Validation and Database Screening}

An internal database was used as a testing set to validate the pharmacophore model. The database included 970 inactive molecules and 30 known inhibitors with experimental activity. To validate the ability of the model to distinguish the active from inactive molecules, the pharmacophore model was used as a 3D query to perform virtual database searching. Some valuable parameters such as total hits $(H t)$, active hits $(\mathrm{Ha}), \%$ yield of actives, \% ratio of actives, enrichment factor $(E)$, and goodness-of-hit score $(G H)$ were calculated (Table 2).

Table 2. Pharmacophore model validation using goodness-of-hit score (GH) score method.

\begin{tabular}{ccc}
\hline Serial No. & Parameter & Pharmacophore Model \\
\hline 1 & Total molecules in database $(\mathrm{D})$ & 1000 \\
2 & Total number of actives in database $(A)$ & 30 \\
3 & Total hits $(H t)$ & 36 \\
4 & Active hits $(H a)$ & 26 \\
5 & $\%$ Yield of actives $[(H a / H t) \times 100]$ & $72 \%$ \\
6 & $\%$ Ratio of actives $[(H a / A) \times 100]$ & $87 \%$ \\
7 & Enrichment factor $(E)[(H a \times D) /(H t \times A)]$ & 24 \\
8 & False negatives $[A-H a]$ & 4 \\
9 & False positives $[H t-H a]$ & 10 \\
10 & Goodness of hit score $(\mathrm{GH}){ }^{\text {a }}$ & 0.75 \\
\hline
\end{tabular}

${ }^{\mathrm{a}}(H a(3 A+H t) / 4 H t A)(1-(H t-H a) /(D-A)) ; G H$ score of $0.7-0.8$ indicates a very good model.

The higher the $E$ value, the greater the ability of a model in identifying the active compounds. The $E$ value for the model was 24 as it had identified 26 active hits from 36 screened compounds, suggesting that the model had a good ability to distinguish the active molecules from the inactive ones. A GH score of $0.7-0.8$ indicates a very good model. It was observed to be 0.75 for the pharmacophore model. These validated results indicate that the model was very efficient for database screening.

Figure 2 shows the virtual screen scheme used in this study. Firstly, the 202,919 molecules in the Specs database were filtered by using Lipinski's rule for the refinement of drug likeness. The model was then used as a filtrating tool in virtual screening to identify potential hits from 168,911 drug-like compounds. Based on a root of the mean square distance (RMSD) value less than $1 \AA$, the 3135 selected molecules were docked into the colchicine-binding site in tubulin. According to the calculation of docking score and interaction analysis, 5 compounds, termed as hits 1-5, were finally selected for further biological evaluation (Table 3). Figure 3 depicts a good pharmacophore mapping of 5 hits on the model. 


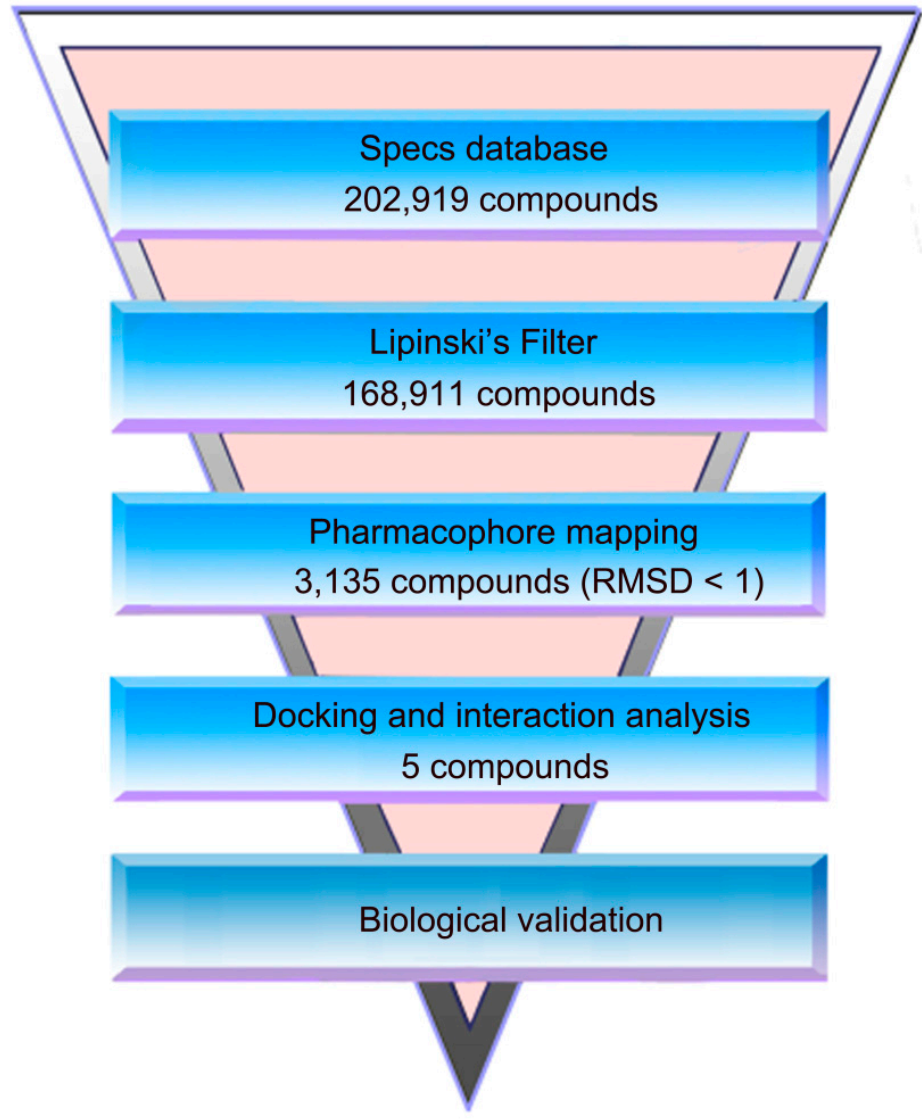

Figure 2. A workflow overview of pharmacophore modeling, selection of compounds, and biological testing.

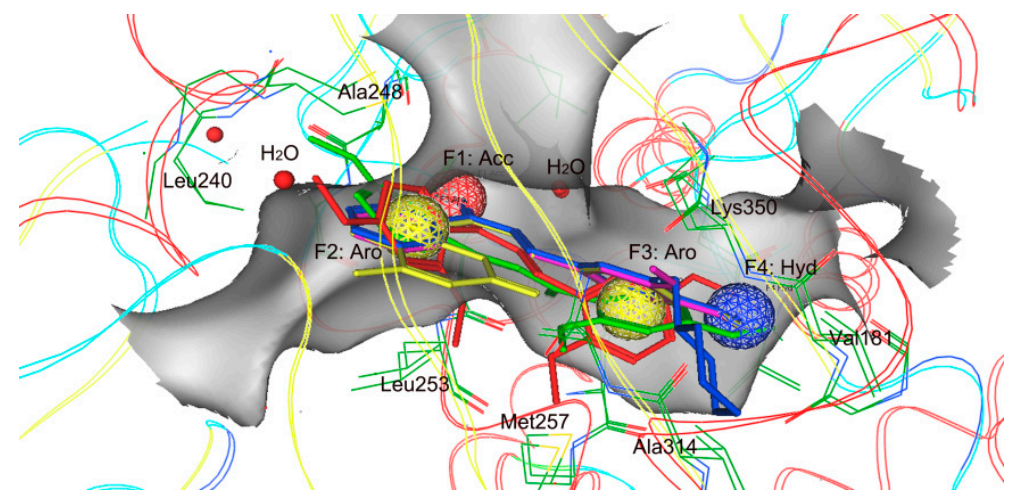

Figure 3. Pharmacophore mapping of five hits on model. Pharmacophore features are color-coded: Red, one hydrogen-bond acceptor feature (F1: Acc); yellow, aromatic features (F2 and F3: Aro); blue, one hydrophobic feature (F4: Hyd). The hits are shown in stick form; protein backbone and active site residues (green) are shown in line form; red balls mean water molecules; a gray pocket represents the shape of the binding site in tubulin. 
Table 3. Hit compounds selected from Specs database.

\begin{tabular}{|c|c|c|c|c|}
\hline Hits & ID Number & Structure & $\operatorname{RMSD}[\AA ̊]^{a}$ & Docking Score $\left[\mathrm{kcal} \cdot \mathrm{mol}^{-1}\right]^{\mathrm{b}}$ \\
\hline 1 & AG-690/11549747 & & 0.5949 & -13.9247 \\
\hline 2 & AH-487/40716190 & & 0.6174 & -13.3812 \\
\hline 3 & AQ-090/41836624 & & 0.6168 & -13.2506 \\
\hline 4 & AN-829/40763420 & & 0.5961 & -13.7928 \\
\hline 5 & AN-829/40458057 & & 0.5974 & -13.4773 \\
\hline
\end{tabular}

\footnotetext{
a The root of the mean square distance between the query features and their matching ligand annotation points (lower RMSD values indicate better mapping of query features and the ligand annotation points); ${ }^{\mathrm{b}}$ Binding free energy between tubulin and a ligand (lower values indicate better binding affinity).
}

\subsection{Biological Activities of Retrieved Molecules}

To further investigate the antiproliferative activity of hits 1-5 against five tumor cells including MCF-7, U87MG, HCT-116, HepG2, and MDA-MB-231, 3-(4,5-dimethylthiazol-2-yl)-2,5-diphenyltetrazolium bromide (MTT) assay was performed. The results revealed that all the hits at the concentration of $40 \mu \mathrm{M}$ had an inhibition rate of more than $50 \%$ (Figure 4). Particularly, hit 1, as the most potent inhibitor, exhibited inhibition rates of more than $80 \%$ against all five tumor cells including MCF-7, U87MG, HCT-116, HepG2, and MDA-MB-231. These results indicated that hit 1 could be developed as an effective anticancer drug with a broad spectrum of anticancer activity.

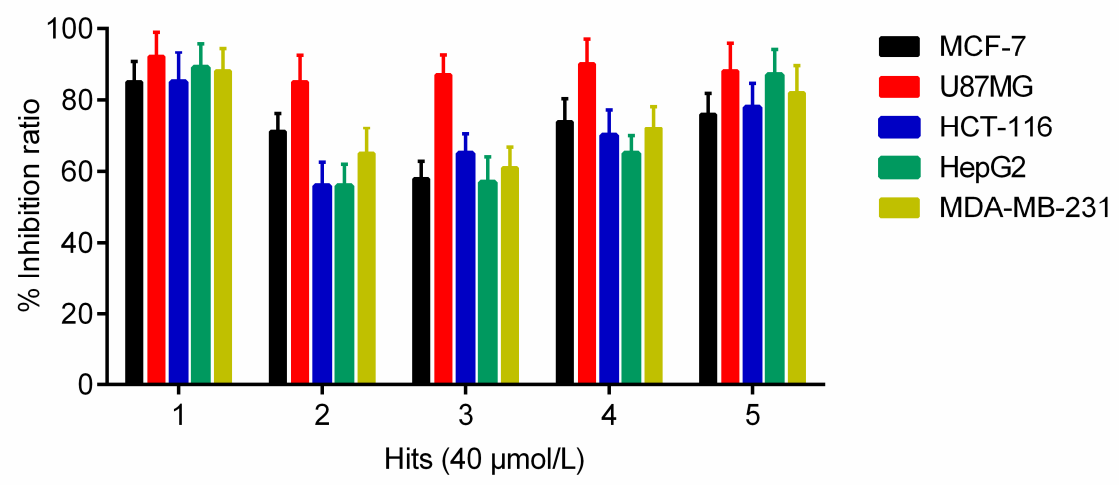

Figure 4. Growth inhibition effects of selected hits 1-5 on five tumor cells (MCF-7, U87MG, HCT-116, HepG2, and MDA-MB-231). The results are representative of three independent experiments and are expressed as mean \pm SD. 


\subsection{Effect of Hit 1 on Tubulin Polymerization and $\left[{ }^{3} \mathrm{H}\right]$ Colchicine Binding}

To further explore the mechanism of action of hit 1 , tubulin polymerization and $\left[{ }^{3} \mathrm{H}\right]$ colchicine binding inhibition assays were performed. A known tubulin inhibitor, combretastatin A-4 (CA-4), was used as the positive control. As shown in Table 4, hit $1\left(\mathrm{IC}_{50}=3.7 \pm 0.5 \mu \mathrm{M}\right)$ showed significant inhibition of tubulin polymerization close to that of the positive control drug CA-4 $\left(\mathrm{IC}_{50}=3.3 \pm 0.6 \mu \mathrm{M}\right)$. In addition, hit 1 showed a $91 \%$ inhibition of $\left[{ }^{3} \mathrm{H}\right]$ colchicine binding at a $20 \mu \mathrm{M}$ concentration. In order to further predict a reasonable binding mode, hit 1 was docked into the colchicine-binding site of tubulin. As shown in Figure 5, hit 1 forms hydrogen-bonding interactions with Ala180 and $\mathrm{H}_{2} \mathrm{O}$. In addition, hit 1 was engaged in a strong hydrophobic interaction with some key amino acids, including Leu248, Ile318, Ala250, Ala316, Leu255, Lys352, Val181, and Ala180, which was crucial for the inhibitor binding of the tubulin. These results show that hit 1 was a potent tubulin inhibitor and could be used as a promising anti-tumor agent.

Table 4. Inhibition of tubulin polymerization and $\left[{ }^{3} \mathrm{H}\right]$ colchicine binding inhibition.

\begin{tabular}{cccc}
\hline Hits & ID Number & Tubulin $\mathrm{IC}_{\mathbf{5 0}}[\mu \mathbf{M}]^{\mathbf{a}}$ & $\left.{ }^{3} \mathbf{H}\right]$ Colchicine Binding Inhibition (\% \pm SD) \\
\hline 1 & AG-690/11549747 & $3.7 \pm 0.5$ & $91 \pm 5.5$ \\
CA-4 & & $3.3 \pm 0.6$ & $96 \pm 3.1$ \\
\hline
\end{tabular}

${ }^{a}$ Inhibition of tubulin polymerization. The tubulin concentration was $10 \mu \mathrm{M} .{ }^{\mathrm{b}}$ Inhibition of colchicine binding. The compound concentration was $20 \mu \mathrm{M}$. The results are representative of three independent experiments and are expressed as mean $\pm \mathrm{SD}$.

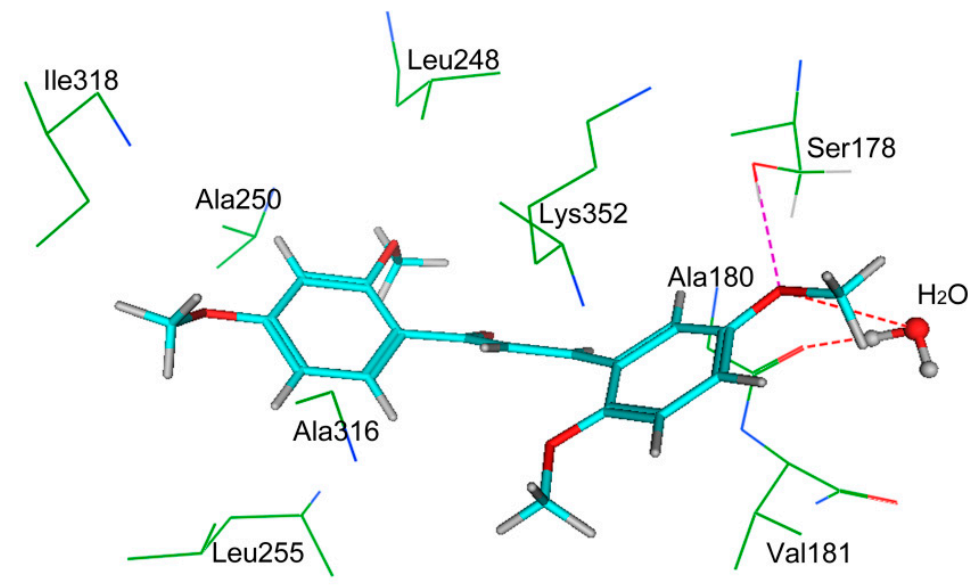

Figure 5. The three-dimensional (3D) ligand-protein interaction diagram for the binding site of tubulin (PDB ID: 6F7C) with hit 1. The active site residues and water molecules are shown in stick form. The hydrogen-bond network with protein residues is represented as red dotted lines. Hit 1 is color-coded as cyan.

\section{Materials and Methods}

\subsection{Pharmacophore Model Generation}

Four X-ray crystallographic structures of the tubulin domain with a high resolution were obtained from the Protein Data Bank (PDB) database (Table 1). These structures were firstly preprocessed and used for the generation of pharmacophore models. Hydrogen was added, Gasteiger partial charges were computed, and then energy minimization was carried out using the Merck molecular force field $94 \times$ (MMFF94 $\times$ ) forcefield [30]. Based on these preprocessed crystal structures, the pharmacophore generation protocol of the Molecular Operating Environment (MOE) (Chemical Computing Group Inc., Montreal, Quebec, Canada) was applied to generate the most representative features of the tubulin 
active site, which are indicated as spheres that represent the essential interaction points with key residues on the ligand binding of the tubulin.

\subsection{Pharmacophore Model Evaluation}

An internal database was constructed with a total of 1000 compounds with 30 actives collected from the reported literature [9,31-33]. The database was used to evaluate the discriminative ability of the pharmacophore model in distinguishing active compounds from the inactive compounds. The database screening was performed using the pharmacophore search protocol available in MOE. The Gunner-Henry (GH) scoring method was applied to quantify the model selectivity precision of hits and the recall of actives from a dataset containing known actives and inactives. This method includes the total hits (Ht), \% yield of actives, \% ratio of actives, enrichment factor (E), false negatives, false positives, and goodness-of-hit score (GH), which were calculated [34]. The GH score ranges from 0 to 1 , which indicates a null model and an ideal model, respectively.

\subsection{Virtual Screening}

To find effective anti-cancer drugs with diverse scaffolds, the Specs database containing more than 200,000 molecules was used for virtual screening because of their structural diversities [35]. Currently, all the compounds of the commercially available Specs database are two-dimensional (2D) planar structures. Therefore, before virtual screening, every compound in the Specs database needs to be transformed into a three-dimensional (3D) structure. The Energy Minimize application in MOE performs potential energy minimization on each molecule in the Specs database. All planar structures were minimized in MOE using the MMFF94x force field, until a root-mean-square gradient of $0.01 \mathrm{kcal}$ $\mathrm{mol}^{-1}$ was reached. In addition, all hydrogen was initially added and the forcefield partial charges computed. In the first screening, Lipinski's rule derived from the statistics of oral drugs was used to find drug-like molecules from the Specs database, because of unique structural characteristics of the colchicines-binding site. Then, based on the established pharmacophore model, the pharmacophore search protocol of the MOE was used to screen hits from these drug-like molecules. According to the manual of the MOE software [36], RMSD means the root of the mean square distance between the query features of the model and their matching ligand annotation points. The matched conformer for each ligand in the database is listed by sorting on a key RMSD. In MOE, lower RMSD values indicate better mapping of ligand annotation points and the query features; better mapping indicates that the ligand has a better binding affinity to its target. An RMSD value of 0 indicates a perfect mapping between the features of the model and their matching ligand annotation points. Based on a RMSD value less than $1 \AA$, the selected hits were used for molecular docking studies.

\subsection{Structure-Based Molecular Docking}

The MOE program was used to perform various steps involved in the docking simulation. The screening hits with a RMSD value of less than $1 \AA$ were docked into the tubulin active site by means of the default triangle matcher algorithm. The dG docking scoring function of MOE estimates the binding free energy between tubulin and a ligand (lower values indicate a better binding affinity) [36,37]. Based on the binding free energies, the final hits were chosen for in vitro evaluation.

\subsection{Cell Proliferation Inhibition Assay}

Cancer cells $\left(5 \times 10^{3}\right.$ cells/well) were seeded in 96-well culture plates (Coring) and incubated overnight. Then, cells were exposed to $40 \mu \mathrm{M}$ of inhibitors and incubated at $37^{\circ} \mathrm{C}$ for $48 \mathrm{~h}$. After that, MTT stock solution $(0.5 \mathrm{mg} / \mathrm{mL})$ was added into each well and cultured for an additional $4 \mathrm{~h}$. The MTT-treated cells were fixed with $150 \mu \mathrm{L}$ of dimethylsulfoxide (DMSO). The absorbance in each individual well was measured at $490 \mathrm{~nm}$ on a microplate spectrophotometer. All assays were performed in triplicate. 


\subsection{Tubulin Polymerization}

According to a previously reported method [38], at $350 \mathrm{~nm}$, tubulin polymerization was turbidimetrically followed in the Beckman model spectrophotometers equipped with electronic temperature controllers. The tubulin concentration was $10 \mu \mathrm{M}$. All assays were performed in triplicate.

\section{7. $\left[{ }^{3} \mathrm{H}\right]$ Colchicine Binding Assay}

According to a previously reported method [39], the binding of $\left[{ }^{3} \mathrm{H}\right]$ colchicines to tubulin was measured. The tubulin and $\left[{ }^{3} \mathrm{H}\right]$ colchicine concentrations were 1.0 and $5.0 \mu \mathrm{M}$, respectively. Compounds were tested at $20 \mu \mathrm{M}$. All assays were performed in triplicate.

\section{Conclusions}

In conclusion, we have successfully constructed an integrated protocol that combines drug-likeness analysis, pharmacophore mapping, and molecular docking studies. Biological validation revealed that five hits identified by the protocol had obvious inhibitory effects on five cancer cells (MCF-7, U87MG, HCT-116, MDA-MB-231, and HepG2), with an inhibition rate of more than $50 \%$ at a concentration of $40 \mu \mathrm{M}$. Our results demonstrated that this integrated protocol can be used as a 3D query to efficiently identify diverse active compounds prior to biological testing, suggesting a great potential for anticancer drug discovery. It is possible that the searching of other commercial databases might find more potential active inhibitors.

Author Contributions: Conceptualization, M.-M.N.; methodology, Y.Z.; software, Y.Z.; validation, M.-M.N.; formal analysis, Y.Z.; investigation, Y.Z.; resources, Y.Z.; data curation, B.D.; writing-original-draft preparation, M.-M.N.; writing review and editing, M.-M.N. and B.D.; visualization, M.-M.N.; supervision, M.-M.N.; project administration, M.-M.N.; funding acquisition, M.-M.N. and B.D.

Funding: This work was financially supported by the National Natural Science Foundation of China (81703419), the Natural Science Foundation of Jiangsu Province (BK20170734), and the Fundamental Research Funds for the Central Universities (2016ZPT005).

Conflicts of Interest: The authors declare no conflict of interest.

\section{References}

1. Bray, F.; Ferlay, J.; Soerjomataram, I.; Siegel, R.L.; Torre, L.A.; Jemal, A. Global cancer statistics 2018: GLOBOCAN estimates of incidence and mortality worldwide for 36 cancers in 185 countries. CA-Cancer J. Clin. 2018, 68, 394-424. [CrossRef] [PubMed]

2. Kerssemakers, J.W.; Munteanu, E.L.; Laan, L.; Noetzel, T.L.; Janson, M.E.; Dogterom, M. Assembly dynamics of microtubules at molecular resolution. Nature 2006, 442, 709. [CrossRef] [PubMed]

3. Chen, X.; Wang, S.M.; Kumar, G.B.; Bare, G.A.; Leng, J.; Bukhari, S.N.; Qin, H.L. Recent developments on phenstatins as potent antimitotic agents. Curr. Med. Chem. 2018, 25, 2329-2352. [CrossRef] [PubMed]

4. Mollinedo, F.; Gajate, C. Microtubules, microtubule-interfering agents and apoptosis. Apoptosis 2003, 8, 413-450. [CrossRef] [PubMed]

5. Checchi, P.M.; Nettles, J.H.; Zhou, J.; Snyder, J.P.; Joshi, H.C. Microtubule-interacting drugs for cancer treatment. Trends Pharm. Sci. 2003, 24, 361-365. [CrossRef]

6. Zhou, J.; Liu, M.; Luthra, R.; Jones, J.; Aneja, R.; Chandra, R.; Tekmal, R.R.; Joshi, H.C. EM012, a microtubule-interfering agent, inhibits the progression of multidrug-resistant human ovarian cancer both in cultured cells and in athymic nude mice. Cancer Chemother. Pharm. 2005, 55, 461-465. [CrossRef] [PubMed]

7. Kavallaris, M. Microtubules and resistance to tubulin-binding agents. Nat. Rev. Cancer 2010, 10, 194. [CrossRef] [PubMed]

8. Dorléans, A.; Gigant, B.; Ravelli, R.B.; Mailliet, P.; Mikol, V.; Knossow, M. Variations in the colchicine-binding domain provide insight into the structural switch of tubulin. Proc. Natl. Acad. Sci. USA 2009, 106, 13775-13779. [CrossRef] [PubMed]

9. Lu, Y.; Chen, J.; Xiao, M.; Li, W.; Miller, D.D. An overview of tubulin inhibitors that interact with the colchicine binding site. Pharm. Res. 2012, 29, 2943-2971. [CrossRef] 
10. Jordan, M.A.; Wilson, L. Microtubules as a target for anticancer drugs. Nat. Rev. Cancer 2004, 4, 253. [CrossRef]

11. Tong, Y.G.; Zhang, X.W.; Geng, M.Y.; Yue, J.M.; Xin, X.L.; Tian, F.; Shen, X.; Tong, L.J.; Li, M.H.; Zhang, C.; et al. Pseudolarix acid $\mathrm{B}$, a new tubulin-binding agent, inhibits angiogenesis by interacting with a novel binding site on tubulin. Mol. Pharm. 2006, 69, 1226-1233. [CrossRef] [PubMed]

12. Ren, X.; Dai, M.; Lin, L.P.; Li, P.K.; Ding, J. Anti-angiogenic and vascular disrupting effects of C9, a new microtubule-depolymerizing agent. Br. J. Pharm. 2009, 156, 1228-1238. [CrossRef] [PubMed]

13. Dark, G.G.; Hill, S.A.; Prise, V.E.; Tozer, G.M.; Pettit, G.R.; Chaplin, D.J. Combretastatin A-4, an agent that displays potent and selective toxicity toward tumor vasculature. Cancer Res. 1997, 57, 1829-1834. [PubMed]

14. Porcù, E.; Viola, G.; Bortolozzi, R.; Persano, L.; Mitola, S.; Ronca, R.; Presta, M.; Romagnoli, R.; Baraldi, P.G.; Basso, G. TR-644 a novel potent tubulin binding agent induces impairment of endothelial cells function and inhibits angiogenesis. Angiogenesis 2013, 16, 647-662. [CrossRef] [PubMed]

15. Brindisi, M.; Ulivieri, C.; Alfano, G.; Gemma, S.; de Asís Balaguer, F.; Khan, T.; Grillo, A.; Chemi, G.; Menchon, G.; Prota, A.E.; et al. Structure-activity relationships, biological evaluation and structural studies of novel pyrrolonaphthoxazepines as antitumor agents. Eur. J. Med. Chem. 2019, 162, 290-320. [CrossRef] [PubMed]

16. Fu, D.J.; Li, P.; Wu, B.W.; Cui, X.X.; Zhao, C.B.; Zhang, S.Y. Molecular diversity of trimethoxyphenyl-1, 2, 3-triazole hybrids as novel colchicine site tubulin polymerization inhibitors. Eur. J. Med. Chem. 2019, 165, 309-322. [CrossRef]

17. Romagnoli, R.; Oliva, P.; Salvador, M.K.; Camacho, M.E.; Padroni, C.; Brancale, A.; Ferla, S.; Hamel, E.; Ronca, R.; Grillo, E.; et al. Design, synthesis and biological evaluation of novel vicinal diaryl-substituted $1 \mathrm{H}-$ Pyrazole analogues of combretastatin A-4 as highly potent tubulin polymerization inhibitors. Eur. J. Med. Chem. 2019, 181, 111577. [CrossRef] [PubMed]

18. Niu, H.; Strecker, T.E.; Gerberich, J.L.; Campbell, J.; Saha, D.; Mondal, D.; Hamel, E.; Chaplin, D.J.; Mason, R.P.; Trawick, M.L.; et al. Structure guided design, synthesis, and biological evaluation of novel benzosuberene analogues as inhibitors of tubulin polymerization. J. Med. Chem. 2019, 62, 5594-5615. [CrossRef]

19. Lin, M.S.; Hong, T.M.; Chou, T.H.; Yang, S.C.; Chung, W.C.; Weng, C.W.; Tsai, M.L.; Cheng, T.R.; Chen, J.J.W.; Lee, T.C.; et al. 4(1H)-quinolone derivatives overcome acquired resistance to anti-microtubule agents by targeting the colchicine site of $\beta$-tubulin. Eur. J. Med. Chem. 2019, 181, 111584. [CrossRef]

20. Jiang, J.; Zhang, H.; Wang, C.; Zhang, Q.; Fang, S.; Zhou, R.; Hu, J.; Zhu, J.; Zhou, Y.; Luo, C.; et al. 1-Phenyl-dihydrobenzoindazoles as novel colchicine site inhibitors: Structural basis and antitumor efficacy. Eur. J. Med. Chem. 2019, 177, 448-456. [CrossRef]

21. Finkelstein, Y.; Aks, S.E.; Hutson, J.R.; Juurlink, D.N.; Nguyen, P.; Dubnov-Raz, G.; Pollak, U.; Koren, G.; Bentur, Y. Colchicine poisoning: The dark side of an ancient drug. Clin. Toxicol. 2010, 48, 407-414. [CrossRef] [PubMed]

22. Niel, E.; Scherrmann, J.M. Colchicine today. Jt. BoneSpine 2006, 73, 672-678. [CrossRef] [PubMed]

23. Ataş, B.; Caksen, H.; Tuncer, O.; Kirimi, E.; Akgün, C.; Odabaş, D. Four children with colchicine poisoning. Hum. Exp. Toxicol. 2004, 23, 353-356. [CrossRef] [PubMed]

24. Subbiah, I.M.; Lenihan, D.J.; Tsimberidou, A.M. Cardiovascular toxicity profiles of vascular-disrupting agents. Oncologist 2011, 16, 1120-1130. [CrossRef] [PubMed]

25. Larocque, K.; Ovadje, P.; Djurdjevic, S.; Mehdi, M.; Green, J.; Pandey, S. Novel analogue of colchicine induces selective pro-death autophagy and necrosis in human cancer cells. PLoS ONE 2014, 9, e87064. [CrossRef] [PubMed]

26. Gould, S.; Westwood, F.R.; Curwen, J.O.; Ashton, S.E.; Roberts, D.W.; Lovick, S.C.; Ryan, A.J. Effect of pretreatment with atenolol and nifedipine on ZD6126-induced cardiac toxicity in rats. J. Natl. Cancer Inst. 2007, 99, 1724-1728. [CrossRef] [PubMed]

27. Granata, R.; Locati, L.D.; Licitra, L. Fosbretabulin for the treatment of anaplastic thyroid cancer. Future Oncol. 2014, 10, 2015-2021. [CrossRef]

28. Hollebecque, A.; Massard, C.; Soria, J.C. Vascular disrupting agents: A delicate balance between efficacy and side effects. Curr. Opin. Oncol. 2012, 24, 305-315. [CrossRef]

29. Prinz, H. Recent advances in the field of tubulin polymerization inhibitors. Expert Rev. Anticancer 2002, 2, 695-708. [CrossRef] 
30. Halgren, T.A. Merck molecular force field. I. Basis, form, scope, parameterization, and performance of MMFF94. J. Comput. Chem. 1996, 17, 490-519. [CrossRef]

31. Chen, J.; Liu, T.; Dong, X.; Hu, Y. Recent development and SAR analysis of colchicine binding site inhibitors. Mini-Rev. Med. Chem. 2009, 9, 1174-1190. [CrossRef]

32. Wang, Y.; Zhang, H.; Gigant, B.; Yu, Y.; Wu, Y.; Chen, X.; Lai, Q.; Yang, Z.; Chen, Q.; Yang, J. Structures of a diverse set of colchicine binding site inhibitors in complex with tubulin provide a rationale for drug discovery. Febs J. 2016, 283, 102-111. [CrossRef] [PubMed]

33. Nguyen, T.L.; McGrath, C.; Hermone, A.R.; Burnett, J.C.; Zaharevitz, D.W.; Day, B.W.; Wipf, P.; Hamel, E.; Gussio, R. A common pharmacophore for a diverse set of colchicine site inhibitors using a structure-based approach. J. Med. Chem. 2005, 48, 6107-6116. [CrossRef] [PubMed]

34. Niu, M.; Wang, F.; Li, F.; Dong, Y.; Gu, Y. Establishment of a screening protocol for identification of aminopeptidase $\mathrm{N}$ inhibitors. J. Taiwan Inst. Chem. Eng. 2015, 49, 19-26. [CrossRef]

35. Qin, T.; Chen, F.; Zhuo, X.; Guo, X.; Yun, T.; Liu, Y.; Zhang, C.; Lai, L. Discovery of novel polo-like kinase 1 polo-box domain inhibitors to induce mitotic arrest in tumor cells. J. Med. Chem. 2016, 59, 7089-7096. [CrossRef] [PubMed]

36. Manual of molecular operating environment (MOE), Version 2007.09; Chemical Computing Group Inc.: Montreal, QC, Canada, 2007.

37. Ul Qamar, T.; Mumtaz, A.; Ashfaq, U.A.; Azhar, S.; Fatima, T.; Hassan, M.; Hussain, S.S.; Akram, W.; Idrees, $\mathrm{S}$. Computer aided screening of phytochemicals from Garcinia against the dengue NS2B/NS3 protease. Bioinformation 2014, 10, 115. [CrossRef] [PubMed]

38. Hamel, E. Evaluation of antimitotic agents by quantitative comparisons of their effects on the polymerization of purified tubulin. Cell Biochem. Biophys. 2003, 38, 1-21. [CrossRef]

39. Verdier-Pinard, P.; Lai, J.Y.; Yoo, H.D.; Yu, J.R.; Marquez, B.; Nagle, D.G.; Nambu, M.; White, J.D.; Falck, J.R.; Gerwick, W.H.; et al. Structure-activity analysis of the interactionof curacin A, the potent colchicine site anti-mitotic agent, with tubulinand effects of analogs on the growth of MCF-7 breast cancer cells. Mol. Pharm. 1998, 53, 62-76. [CrossRef]

Sample Availability: Samples of the compounds are available from the authors.

(C) 2019 by the authors. Licensee MDPI, Basel, Switzerland. This article is an open access article distributed under the terms and conditions of the Creative Commons Attribution (CC BY) license (http://creativecommons.org/licenses/by/4.0/). 